\title{
Clasificación PIRO en sepsis grave y shock séptico pediátrico: Nuevo modelo de estratificación y su utilidad en pronóstico
}

\author{
Daniela Arriagada S., Franco Díaz R., Alejandro Donoso F. y Pablo Cruces R.
}

\section{PIRO classification in pediatric severe sepsis and septic shock: A new model for staging and its potential usefulness in prognoses}

Background: Sepsis is a dynamic process that involves complex interactions between the pathogenic microorganisms and the host. The understanding of this heterogeneous disease has led to the development of a new system for stratification of septic patients: the PIRO system: Predisposition (P) -Insult/Infection (I) -Response (R) -Organ disfunction $(\mathrm{O})$, a classification aimed to determine the risk of death in patients with sepsis. Only a few studies have validated this classification system in children. Objective: To empirically test the accuracy of the PIRO system in pediatric patients with septic shock and severe sepsis and associate its individual components to predict mortality. Patients and Method: A retrospective chart review was performed in a 13 bed PICU during 24 months (January 2006 to December 2007) Demographic, clinical and microbiological data were recorded in all patients with a diagnosis of septic shock and severe sepsis during the study period. For all patients the PIRO classification system was applied by one of four authors using paramethers measured at admission. Results: A total of 42 patients were included with a mean age of 11 months (range 3.25-58.3) of which 52\% were male. Overall mortality was $19 \%$ and variables associated with mortality for each category were: $(\mathrm{P})$ Chronic illness (OR: 7 IC95\% 0.95-51) and Immunodeficiency (OR: 6.2; IC95\% 1.1-35.2); (R) leucopenia (OR 9; IC95\%: 1.96-41.72); (O) more than three dysfunctional organs (OR: 6.1; IC95\%: 1.22-31). None of the (I) variables were associated with mortality. Conclusions: The PIRO classification system identified factors associated with a fatal outcome in our population. The test is relatively simple to apply but cross-sectional studies are required to define variables associated with death that should then be prospectivelly validated.

Key words: Infection; sepsis; severe sepsis; septic shock; staging; PIRO model.

Palabras clave: Infección; sepsis; sepsis grave; shock séptico; estratificación, PIRO.

\section{Introducción}

$\mathrm{L}$ a sepsis es la respuesta del hospedero a un agente microbiano o a sus toxinas. En la práctica clínica, este término resume una miríada de condiciones patogénicas de diversa gravedad, para un grupo heterogéneo de pacientes, todo lo cual hace a estos difícil de estratificar adecuadamente.

Tras el esfuerzo de distintas sociedades científicas estadounidenses y luego de más de una década de discusión, en el año 1991 se logró una definición de consenso, la cual fue actualizada en el año 2001 al agregar al debate a diversas sociedades científicas europeas ${ }^{1,2}$. En ésta se incorporó el término síndrome de respuesta inflamatoria sistémica (SRIS) y se definió sepsis, sepsis grave (SG) y shock séptico (SS), terminología de uso habitual en la actualidad. Sin embargo, estos conceptos presentan importantes limitaciones: poco sustento fisiopatológico, escasa especificidad, no permiten una estratificación precisa y carecen de significado pronóstico, por nombrar algunas ${ }^{3,4}$. Debido a su uso masivo, la clasificación de consenso ha sido muy útil para el diagnóstico precoz, y para precisar los criterios de inclusión de numerosos ensayos clínicos de intervenciones terapéuticas, lo que ha permitido conocer mejor la historia natural de la enfermedad ${ }^{5,6}$.

En el año 2000, en forma paralela al anterior, un nuevo sistema de categorización de riesgo fue propuesto en la Quinta Mesa Redonda de Sepsis de Toronto, denominado sistema "IRO", al cual posteriormente se agregó una "P" inicial, acuñando el término conocido en la actualidad como "PIRO", derivado de su acrónimo en inglés "Predisposition", "Insult/Infection”, "Response” y "Organ disfunction" "2,8. Éste valora en forma separada los distintos aspectos dependientes del hospedero como del microorganismo agresor, en un paralelo con el sistema oncológico TNM desarrollado por Denoix, que es capaz de determinar la conducta terapéutica y la sobrevida en las distintas categorías 9 .

Potencialmente esta clasificación puede evaluar diversos ámbitos, como son las condiciones Predisponentes (genéticas, biológicas y culturales), la "Injuria/Infección" (agresión) en el caso de sepsis, la infección (microbiolo-

\author{
Universidad del Desarrollo \\ Clínica Alemana, Santiago, \\ Chile \\ Facultad de Medicina \\ Becada Pediatría (DAS) \\ Becado Programa de Medicina \\ Intensiva Infantil (FDR) \\ Hospital Padre Hurtado, \\ Santiago, Chile \\ Área de Cuidados Críticos (ADF, \\ $P C R)$
}

Recibido: 28 de abril de 2009 Aceptado: 15 de noviembre de 2009

Correspondencia a:

Alejandro Donoso Fuentes adonoso@hurtadohosp.cl 
gía y sitio de la infección), la Respuesta del hospedero (fisiológica, bioquímica y biológica) y el desarrollo de disfunción de Órganos ${ }^{2,10-13}$. De esta forma, nos permitiría categorizar en forma más certera e individual a estos pacientes, pues estamos reconociendo y valorando los distintos predictores independientes de pronóstico para una respuesta séptica.

A pesar de la enorme potencialidad de esta clasificación, las definiciones han permanecido muy teóricas, con escasa literatura y aplicación clínica ${ }^{2,8,14}$.

Objetivo. Evaluar la aplicabilidad de una propuesta inicial de estratificación "PIRO" en SG y SS y la asociación de sus variables con el pronóstico, en una muestra de niños críticamente enfermos.

\section{Pacientes y Métodos}

En forma retrospectiva, se analizaron las historias clínicas de los pacientes bajo 15 años de edad cuyo diagnóstico de egreso fue SS o SG según la Conferencia de Consenso de 1991 del American College of Chest Physicians (ACCP) y de la Society of Critical Care Medicine (SCCM) y las modificaciones propuestas por la Conferencia de
Consenso de la Sepsis Pediátrica del año $2002^{1,2,15}$.

De este modo, se definió SG como la existencia de sepsis más una de las siguientes evidencias de disfunción orgánica: disfunción cardiovascular, síndrome de distress respiratorio agudo (SDRA), o dos o más de otras disfunciones orgánicas (respiratoria, renal, neurológica, hematológica o hepática). SS fue definido como sepsis más la presencia de disfunción cardiovascular, a pesar de la administración de fluidos.

La unidad de cuidados intensivos (UCI) pediátrica del Hospital Padre Hurtado posee 13 camas mixtas y no cuenta con pacientes cardio-quirúrgicos en etapa de post operatorio inmediato. El período analizado comprendió entre enero de 2006 y diciembre de 2007 (24 meses). Se incluyeron a todos los pacientes, independiente de la duración de su estadía intra UCI.

Se consignaron las características demográficas, clínicas, microbiológicas, puntajes de gravedad $\mathrm{PIM}_{2}$ y PELOD y condición de vivo o muerto al egreso ${ }^{16,17}$. Las patologias crónicas analizadas fueron: hemato-oncológica (leucemia), daño pulmonar, cardiopatía congénita, epilepsia, pseudo-obstrucción intestinal crónica, malformaciones congénitas de la pared abdominal y del tracto urinario.

Las variables analizadas fueron clasificadas según la propuesta PIRO (Figura 1).
Figura 1. Variables evaluadas en pacientes con shock séptico y sepsis grave, agrupadas según el sistema PIRO.
P: Predisposición:

Edad del paciente (mayor o menor de un mes)

Presencia de patología crónica (co-morbilidades)*

Factores genéticos (síndrome genético, dismorfias y/o malformaciones)

Condición de inmunodeficiencia

\section{I: Injuria/Infección:}

Foco de la infección (pulmonar, abdominal, urinario, meníngeo)

Existencia de cultivo negativo (hemocultivo). Bacteriemia (-)

Características microbiológicas

\section{R: Respuesta:}

Reactantes de fase aguda (Proteína $C$ reactiva)

Recuento leucocitario disminuido $\left(<5.000\right.$ céls $/ \mathrm{mm}^{3},<3.000$ céls $\left./ \mathrm{mm}^{3}\right)$

\section{O: Disfunción de Órganos:}

Fueron dicotomizados como mayor o menor de tres, según la definición:

- Cardiovascular: refractariedad a $\geq 40 \mathrm{ml} / \mathrm{kg}$ en $1 \mathrm{~h}$ más caída de la PA $<$ p5 para la edad o PAS < 2 DE normal para la edad o necesidad de fármacos vasoactivas (dopamina $>5 \mathrm{mcg} / \mathrm{kg} / \mathrm{min}$ o dobutamina, epinefrina o norepinefrina a cualquier dosis) o (dos de los siguientes hallazgos: acidosis metabólica, hiperlactacidemia, oliguria, Ilene capilar enlentecido, delta temperatura central y periférica $>3{ }^{\circ} \mathrm{C}$

- Pulmonar: hipoxemia arterial, $\mathrm{PaO}_{2} / \mathrm{FiO}_{2}<300$ o necesidad no electiva de $\mathrm{VM}$ invasora

- Neurológico: Escala de Glasgow $\leq 11$ o cambio agudo en el estado mental con descenso del Glasgow > 3

- Renal: incremento creatininemia dos veces sobre el valor superior normal para la edad

- Hepático: hiperbilirrubinemia $\geq 4 \mathrm{mg} / \mathrm{dL}$, no aplicable para recién nacido

- Hematológico: recuento plaquetario $<80.000 / \mathrm{mm}^{3}$, prolongación de INR $>2$

(*) Según definición del Comité de Enfermedades Crónicas en la Infancia, cualquier afección de duración sobre 3 meses y que altera la normal actividad; incluyó condiciones que generaron hospitalización continua de 30 días o más; como también, aquellas con diagnóstico precoz de certeza. 
Estadígrafos utilizados: Las variables continuas son expresadas como medianas e intercuartiles y fueron analizadas con el test de Kruskall Wallis. Se evaluó la asociación de las variables con resultado letal mediante la razón de disparidad (Odds ratio), expresado con su intervalo de confianza (IC 95\%).

\section{Resultados}

En el período examinado egresaron 1.223 pacientes de UCI, 42 (3,5\%) cumplieron los criterios de inclusión, edad 11 meses $(3,25-58,5), 7 \%$ tenían menos de un mes de vida y $55 \%$ eran de sexo masculino.

Los puntajes de PIM $_{2}$ y PELOD fueron 16,6\% (rango $3,1-83 \%$ ) y $18,2 \%$ (rango $0,1-99,6 \%$ ), respectivamente. La mortalidad fue de $19 \%(8 / 42)$; todos los fallecidos tenían más de un mes de vida.

Las características demográficas, puntajes de gravedad y las variables continuas analizadas (recuento de leucocitos plasmáticos y proteína $\mathrm{C}$ reactiva) se muestran en la Tabla 1, agrupados en sobrevivientes y fallecidos.

De los pacientes fallecidos, $50 \%$ de ellos ocurrió dentro de las primeras $24 \mathrm{~h}$ de estadía en UCI, todos ellos con leucopenia. El resto falleció en un rango de cinco a 24 días. Las patologías crónicas asociadas con mortalidad fueron daño pulmonar (n: 2), epilepsia (n: 1), cardiopatía (n: 1) y leucemia (n: 1).

Para el total de pacientes, los focos de infección más frecuentes fueron abdominal (n: 10), pulmonar (n: 10), meníngeo (n: 6), urinario (n: 5) y cutáneo (n: 4). En los fallecidos los sitios más frecuentes fueron pulmonar (n: 3 ) y abdominal (n: 1). Para los pacientes fallecidos dentro de las primeras 24 horas, sólo en uno de cuatro se identificó un foco (pulmonar).

En 27 pacientes (64\%) la etiología fue identificada. Los microorganismos más frecuentemente aislados fueron Streptococcus pneumoniae (n: 7), Escherichia coli (n: 5), Neisseria meningitidis (n: 4), Staphylococcus aureus (n: 3) y Pseudomonas aeruginosa (n: 2).

En $75 \%$ de los pacientes fallecidos se identificó la etiología: $N$. meningitidis (n: 2), P. aeruginosa (n: 1), E. coli (n: 1), S. aureus (n: 1) y S. pneumoniae (n: 1).

La asociación de las variables con mortalidad se observa en la Tabla 2:

- Predisposición $(\mathrm{P})$ : antecedente de patología crónica (OR: 7,0; IC95\% 0,95-51) e inmunodeficiencia (OR: 6,2; IC95\% 1,1-35,16).

- Infección (I): Ninguna de las variables microbiológicas, ni lugar de infección se asoció a mayor mortalidad. Mientras que la presencia de un foco primario fue protector para resultado letal. (OR: 0,27; IC95\% 0,05$1,31)$.

- Respuesta (R): leucopenia $<5.000$ céls $/ \mathrm{mm}^{3}$ (OR 9;
IC95\% 1,96-41,7), siendo mayor al profundizarse a menos de 3.000 céls $/ \mathrm{mm}^{3}$ (OR 11,57; IC95\% 1,98167,9).

- Disfunción de órganos $(\mathrm{O})$ : el desarrollo de disfunción de tres o más órganos (OR: 6,1; IC95\% 1,22-31).

\begin{tabular}{|c|c|c|c|}
\hline & Vivos & Fallecidos & P-value \\
\hline $\mathrm{n}$ & 34 & 8 & \\
\hline Edad (meses) & $10,5(2,8-60)$ & $19(6,5-54)$ & 0,6 \\
\hline Sexo masculino & $55 \%$ & $44 \%$ & 0,42 \\
\hline $\mathrm{PIM}_{2}(\%)$ & $6,2(1,9-11,7)$ & $27,25(0,15-75,5)$ & 0,6 \\
\hline PELOD (\%) & $0,1(0,1-1,7)$ & $97,5(13,5-99,7)$ & 0,017 \\
\hline Leucocitos $/ \mathrm{mm}^{3}$ & $18.150(9.300-25.100)$ & $3.000(2.400-3.400)$ & 0,001 \\
\hline PCR & $141(80-242)$ & $142,5(50-254)$ & 0,88 \\
\hline \multicolumn{4}{|c|}{$\begin{array}{l}\text { PCR: Proteína } C \text { reactiva }(\mathrm{mg} / \mathrm{l}) ; \mathrm{PIM}_{2} \text { : Paediatric Index of mortality. PELOD: Paediatric logistic organ } \\
\text { dysfunction }\end{array}$} \\
\hline
\end{tabular}

Tabla 2. Variables estudiadas categorizadas según sistema PIRO y su asociación con resultado letal, expresadas como Odds ratio (OR) e intervalo de confianza 95\% (IC95\%)

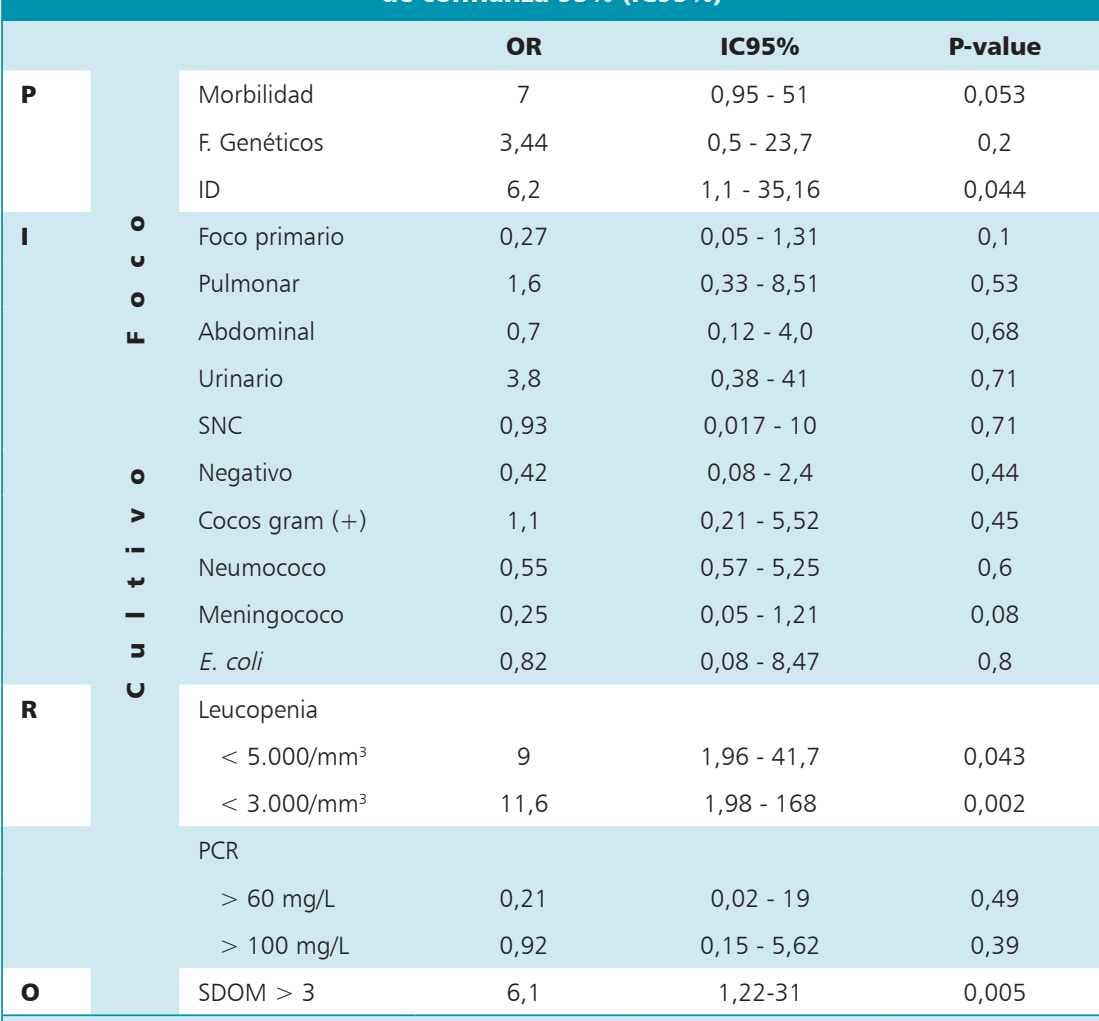

F. Genéticos: Factores Genéticos; SNC: sistema nervioso central; PCR: proteína C reactiva ( $\mathrm{mg} / \mathrm{l})$; SDOM: Síndrome de disfunción orgánica múltiple, ID: Inmunodeficiencia 


\section{Discusión}

La sepsis con sus diagnósticos asociados es un problema de salud trascendental, constituyendo una de las principales causas de mortalidad pediátrica en el mundo y a su vez, implica un elevadísimo consumo de recursos en salud ${ }^{18,20}$.

La mortalidad del SS en la población infantil permanece alrededor de $10 \%$ en países desarrollados; sin embargo, se observan cifras más elevadas en otras realidades, no obstante haber disminuido progresivamente desde la década del 60 hasta la actualidad ${ }^{18,21-23}$.

A pesar de este notable avance en el tratamiento del $\mathrm{SS}$, aparte del control de la infección (antimicrobianos y cirugía cuando se requiera), la terapia es meramente de soporte $^{19}$. El pronóstico del paciente con SS dependerá básicamente de la rapidez con que se instauren las medidas terapéuticas apropiadas ${ }^{19,24-26}$. Pero, más allá de lo eficaces de estas medidas, existen otros aspectos a considerar en forma particular, en búsqueda de factores pronósticos y una terapia orientada individualmente $\mathrm{e}^{7,8,14,15}$.

Desde este punto de vista, la sepsis es un complejo grupo de enfermedades altamente variables, caracterizada por el desarrollo de inflamación, como forma de respuesta sistémica del hospedero ${ }^{27}$. Por lo tanto, idealmente debe ser clasificada en subgrupos más homogéneos ${ }^{8,14,15,27}$, siendo la clasificación PIRO un avance hacia una visión futura de categorización individual ${ }^{8,14}$.

Nuestra serie de pacientes demuestra que es fácil de aplicar una vez definidas las variables a utilizar, permitiendo una categorización más individual. Esto no sólo tiene implicancias pronósticas, sino también permite buscar beneficios de determinadas terapias para grupos específicos de pacientes ${ }^{14,27,28}$.

Como es de esperar, no todas las variables presentaron la misma asociación a resultado letal, careciendo en la actualidad de una sistematización del análisis de todas las variables.

A continuación se comenta a modo esquemático cada una de las categorías examinadas:

Predisposición: En nuestra casuística, la presencia de patología crónica presentó un mayor riesgo de fallecer, lo cual también ha sido corroborado en una reciente comunicación, donde se señaló que la mortalidad en niños con SG y enfermedad crónica era cuatro veces mayor que en niños que cursan con $\mathrm{SG}$, pero previamente sanos $(8 \mathrm{y}$ $2 \%$, respectivamente $)^{20}$. Un especial grupo lo constituyen los pacientes portadores de patología oncológica, cuya mortalidad se podría triplicar en la condición de sepsis, reportándose en algunas series una mortalidad cercana al $50 \%$ cuando desarrollan $\mathrm{SS}^{22,29}$. En esta serie, sólo un paciente tenía patología oncológica; sin embargo, la condición de inmunodeficiencia se asoció en forma potente con exitus letalis.
Cada vez hay más evidencia del rol transcendente que juega la constitución genética del individuo para el pronóstico de la sepsis. En un clásico estudio, Sorensen y cols ${ }^{30}$, cotejaron el riesgo de morir de niños adoptados y la causa de muerte del padre biológico (factor genético) y adoptivo (factor ambiental) concluyendo que el riesgo relativo de morir por una causa infecciosa era casi cinco veces mayor cuando el padre biológico había fallecido por una infección dentro de los primeros cincuenta años de vida. Este riesgo fue aún mayor que la asociación para fallecer por causa oncológica o de enfermedad cardiovascular, cuyo factor hereditario es conocido hace casi medio siglo.

Se han descrito variados defectos genéticos que determinan alteraciones de la inmunidad (déficit del complemento, defecto en neutrófilos, etc), asociando de esta forma los componentes "I" y "R". Por ejemplo, mutaciones de toll like receptor, que impiden al hospedero montar una respuesta inmune $(\mathrm{R})$ al no reconocer al patógeno ${ }^{7,8,27}$. También los defectos pueden ser agente específico (I), por ejemplo el déficit de complemento en meningococcemia recurrente ${ }^{31}$.

No hay que olvidar que existe un gran número de defectos adquiridos en la inmunidad del hospedero que crean condiciones predisponentes para el desarrollo de sepsis (quemaduras, trauma, disrupción de barrera muco/cutánea), las cuales no fueron analizadas para esta casuística, al no presentarlas ningún paciente.

Injuria/Infección: En esta serie descrita, las variables relacionadas con esta categoría no se asociaron a resultado letal. Al revisar la literatura científica frecuentemente se describe que el sitio, tipo y magnitud de la infección que origina la $\mathrm{SG}$ tiene importancia en el pronóstico. Es así como en adultos se observa mayor mortalidad en infecciones pulmonares, intra-abdominales y del sistema nervioso central al compararla con las originadas en piel, tejidos blandos o foco genitourinario ${ }^{13,32}$. Nuestra serie mostró que la presencia de un foco primario fue protectora para resultado letal, concordante con lo descrito en otros estudios $^{33}$.

En forma paralela a la predisposición individual, las características del microorganismo causal son fundamentales, ya que la SG también puede ser resultado de un inóculo enorme de microorganismos que sobrepasen las defensas del hospedero, o bien, una cantidad mínima de microorganismos con virulencia extrema ${ }^{27}$. Es así como la SG por cocáceas grampositivas y hongos se han relacionado con una mayor mortalidad ${ }^{34,35}$. En nuestra casuística no hubo diferencias según las características microbiológicas.

En el análisis se debe considerar que al incorporar a todos los pacientes, independiente de la duración de su permanencia en UCI, incluimos a aquellos con períodos 
de estadía breves (fallecidos dentro de las primeras $24 \mathrm{~h}$ ), en quienes puede tener un mayor impacto la magnitud de la infección (en el modelo aquí propuesto) y por el contrario, la respuesta a la noxa puede no ser evidenciada en toda su magnitud.

Respuesta: Como se ha señalado previamente, el desarrollo por el hospedero de una respuesta inflamatoria es clave en el entendimiento de la SG y SS. En ésta influyen factores como sexo, raza, edad y condición nutricional. Es evidente que existe una sobreposición entre dos componentes del PIRO, ya que los factores predisponentes (P) pueden afectar también la respuesta del hospedero (R). Es conocida la existencia de un modelo caracterizado por una gran respuesta inflamatoria (hiper-inflamación), como la observada en meningococcemia o shock tóxico estreptocóccico, mientras que en el otro extremo tenemos pacientes con una escasa capacidad de montar una respuesta inflamatoria (neutropénico febril o sometido a trasplante de precursores hematopoyéticos) $)^{27,36}$. Ambas condiciones, de una u otra manera, están implicadas en la progresión de la sepsis y un eventual resultado letal.

Diversos bio-marcadores han sido descritos en sepsis, orientados básicamente al diagnóstico precoz, pero su utilidad en el pronóstico es controversial ${ }^{37}$. Viallon y cols, en una reciente comunicación evaluaron el rol de diversos marcadores de la respuesta inflamatoria, encontrando que el mejor de ellos fue la concentración de procalcitonina sérica para la evaluación de la gravedad ${ }^{38}$. Al igual que este y otros reportes, en nuestra casuística la PCR no se relacionó con gravedad, ni pronóstico letal ${ }^{39}$. Otros estudios han encontrado asociación estadística de gravedad con concentraciones plasmáticas de citoquinas y actividad de la proteína $\mathrm{C}$ de la coagulación ${ }^{40-42}$; sin embargo, la temporalidad de sus mediciones aún no está consensuada, siendo su uso limitado.

La potente asociación de leucopenia con exitus letalis llama fuertemente la atención en nuestra casuística, inclusive considerando el bajo número de pacientes con meningococcemia, donde es conocido lo ominoso de este indicador ${ }^{43}$. Probablemente ésta es un reflejo global de una respuesta inadecuada debida a múltiples factores; no obstante, es una herramienta importante para el médico clínico en la aproximación inicial, entregando un parámetro objetivo asociado al riesgo de fallecer.

Disfunción de órganos: El mecanismo exacto de la falla de órganos en sepsis aún es desconocido, siendo descritas múltiples vías que llevan finalmente a una interrupción energética celular y el cese de función ${ }^{36}$. En la serie aquí presentada, la disfunción de tres o más órganos tuvo seis veces más chance de fallecer, similar a lo reportado en otros estudios ${ }^{5,22,44}$. Esto no es de extrañar, pues refleja tanto la magnitud como la duración del daño, ya sea este ocasionado por una agresión directa, disoxia, respuesta inflamatoria desmedida del hospedero y/o falta de respuesta anti-inflamatoria, por nombrar algunas.

La gravedad de la disfunción orgánica puede tener influencia en la estratificación terapéutica. Así, existe evidencia que la neutralización del factor de necrosis tumoral (precoz mediador inflamatorio) es más efectivo en pacientes sin una disfunción orgánica importante ${ }^{7}$, mientras que el uso de drotrecogin a activado es más probable que beneficie a pacientes con compromiso de dos o más órganos y niveles de proteína $\mathrm{C}$ activada bajos ${ }^{42,45}$. A futuro, pudiese jugar un papel en este aspecto la medición dinámica de la respuesta celular a la agresión con indicadores de apoptosis, hipoxia citopática o estrés celular.

Un potencial beneficio del modelo PIRO es el permitir discriminar entre la morbilidad que nace de la infección de aquella originada de la respuesta a la misma. Intervenciones terapéuticas orientadas a una modulación de la respuesta (tratamiento anti-inflamatorio) pueden afectar negativamente la capacidad de contener la infección (tratamiento anti-infeccioso). Por otra parte, tratamientos orientados a la infección, pueden ser de poca utilidad si el impacto en la morbilidad es debido a una respuesta desmedida del hospedero ${ }^{46}$.

Es necesario comentar que nuestra serie presenta limitaciones como el ser de carácter retrospectivo y con un pequeño número de pacientes. Además describe sólo la realidad local de nuestra UCI; no obstante, creemos importante darla a conocer e iniciar la incorporación de una nueva mirada al paciente séptico, la cual nos permitirá re-examinar su fisiopatología como también poder ser útil en futuras investigaciones tanto para población infantil como adulta ${ }^{47}$.

\section{Conclusiones}

En nuestra casuística, la evaluación mediante el modelo PIRO permitió asociar la existencia de co-morbilidad crónica, condición de inmunodeficiencia, presencia de leucopenia y desarrollo de disfunción de tres o más órganos, con un mayor riesgo de mortalidad.

A futuro, el desarrollo de una propuesta de categorización como la aquí reportada, para una población y patología muy heterogénea, permitirá una mejor caracterización de este síndrome en base a una evaluación conjunta de los factores predisponentes, el tipo de infección subyacente, las características de la respuesta del hospedero y la magnitud de la disfunción orgánica resultante.

El sistema de clasificación PIRO es útil y fácil de implementar, pero aún se mantiene en forma conceptual. Es importante realizar estudios transversales multicéntricos para definir una estratificación PIRO consensuada para 
pediatría y luego validarla en forma prospectiva para precisar su utilidad en el diagnóstico y evaluación del riesgo.

\section{Resumen}

Introducción: La compresión de la sepsis como un proceso dinámico, resultado de la interacción entre hospedero y agente infeccioso, ha llevado al sistema de estratificación "PIRO” (P) Predisposición, (I) Injuria/ Infección, (R) Respuesta y $(\mathrm{O})$ disfunción de Órganos, clasificación orientada a predecir la muerte en pacientes con sepsis, a ganar adeptos. Sin embargo, faltan estudios clínicos que lo validen. Objetivo: Evaluar la certeza de la clasificación "PIRO" en sepsis grave y shock séptico para predecir mortalidad. Pacientes y Método: Estudio retrospectivo efectuado en una UCI pediátrica de 13 camas durante 24 meses (enero 2006 a diciembre 2007). Uno de los cuatro autores registró las características demográficas, clínicas y microbiológicas de la totalidad de pacientes ingresados con diagnóstico de sepsis grave y shock séptico, agrupándolos según sobrevida. Fueron clasificadas estas variables según sistema PIRO Se evaluó la asociación de estas variables con la mortalidad. Resultados: 42 pacientes, edad 11 meses $(3,2-58)$ y mortalidad $19 \%$. Las variables asociadas a mortalidad fueron: (P) antecedente de patología crónica (OR: 7; IC95\% 0,95$51)$ e inmunodeficiencia $(6,2 ; 1,1-35,2)$; (R) leucopenia (9; 1,96-41,72); (O) disfunción de 3 o más órganos $(6,1$; 1,22-31). Ninguna de las variables (I) se asoció a mortalidad. Conclusiones: El sistema "PIRO" es un modelo en desarrollo para una clasificación individual, de fácil aplicación. Permite reconocer factores asociados a un resultado fatal, en la presente casuística dado por inmunodeficiencia, leucopenia y fallo de tres o más sistemas. Es importante realizar estudios transversales para definir una etapificación PIRO consensuada y luego validarla prospectivamente.

\section{Referencias}

1.- American College of Chest Physicians/Society of Critical Care Medicine Consensus Conference: Definitions of sepsis and multiple organ failure and guidelines for the use of innovative therapies in sepsis. Crit Care Med 1992; 20: 864-74.

2.- Levy M M, Fink M P, Marshall J C, Abraham E, Angus D, Cook D, et al. 2001 SCCM/ESICM/ ACP/ATS/SIS International Sepsis Definitions Conference. Intensive Care Med 2003; 29: 5308.

3.- Vincent J L. Dear SIRS, I'm sorry to say that I don't like you. Crit Care Med 1997; 25: 372-4.

4.- Carlet J, Cohen J, Calandra T, Opal S M, Masur H. Sepsis: time to reconsider the concept. Crit Care Med 2008; 36: 964-6.

5.- Goldstein B, Nadel S, Peters M, Barton R, Machado F, Levy H, et al. ENHANCE: results of a global open-label trial of drotrecogin alfa (activated) in children with severe sepsis. Pediatr Crit Care Med 2006; 7: 200-11.

6.- Sprung C, Annane D, Keh D, Moreno R, Singer M, Freivogel K, et al. Hydrocortisone therapy for patients with septic shock. N Engl J Med 2008; 358: 111-24.

7.- Marshall J C, Vincent J L, Fink M P, Cook D J, Rubenfeld G, Foster D, et al. Measures, markers, and mediators: Toward a staging system for clinical sepsis. A Report of the Fifth Toronto Sepsis Roundtable, Toronto, Ontario, Canada, 25-26 October, 2000. Crit Care Med 2003; 31: $1560-7$.

8.- Opal S M. Concept of PIRO as a new conceptual framework to understand sepsis. Pediatr Crit Care Med 2005; 6: S55-S60.
9.- Denoix P X. Enquete permanent dans les centres anticancereaux. Bull Inst Natl Hyg 1946; 1: 70 5.

10.- Vincent J L, Wendon J, Groeneveld J, Marshall J C, Streat S, Carlet J. The PIRO concept: $\mathrm{O}$ is for organ dysfunction. Crit Care 2003; 7: 260-4.

11.- Angus D C, Burgner D, Wunderink R, Mira J P, Gerlach H, Wiedermann C J, et al. The PIRO concept: $\mathrm{P}$ is for predisposition. Crit Care 2003; 7: 248-51.

12.- Gerlach H, Dhainaut J F, Harbarth S, Reinhart K, Marshall J C, Levy M. The PIRO concept: $\mathrm{R}$ is for response. Crit Care 2003; 7 : 256-9.

13.- Vincent J L, Opal S, Torres A, Bonten M, Cohen J, Wunderink R. The PIRO concept: I is for infection. Crit Care 2003; 7: 252-5.

14.- Moreno R P, Metnitz B, Adler L, Hoechtl A, Bauer P, Metnitz P G, et al. Sepsis mortality prediction based on predisposition, infection and response. Intensive Care Med 2008; 34: 496-504.

15.- Goldstein B, Giroir B, Randolph A. International Consensus Conference on Pediatric Sepsis. Pediatr Crit Care Med 2005; 6: 2-8.

16.- Slater A, Shann F, Pearson G. PIM2: A revised version of the Paediatric Index of Mortality. Intensive Care Med 2003; 29: 278-85.

17.- Leteurtre $S$, Martinot A, Duhamel A, Proulx F, Grandbastien B, Cotting J, et al. Validation of the paediatric logistic organ dysfunction (PELOD) score: prospective, observational, multicentre study. Lancet 2003; 362: 192-7.

18.- Watson R S, Carcillo J A, Linde-Zwirble W T, Clermont G, Lidicker J, Angus D C. The epidemiology of severe sepsis in children in the
United States. Am J of Respiratory Crit Care Med 2003; 167: 695-701.

19.- Dellinger R P, Carlet J M, Masur H, Gerlach H, Calandra T, Cohen J, et al. Surviving sepsis campaign guidelines for management of severe sepsis and septic shock. Crit Care Med 2004; 32: 858-71.

20.- Odetola F O, Gebremariam A, Freed G L. Patient and hospital correlates of clinical outcomes and resource utilization in severe pediatric sepsis. Pediatrics 2007; 119: 487-94.

21.- Proulx F, Fayon M, Farrel CA, Lacroix J, Gauthier M. Epidemiology of sepsis and multiple organ dysfunction syndrome in children. Chest 1996; 109: 1033-7.

22.- Wolfler A, Silvani P, Musicco M, Musicco A, Antonelli M. Incidence of and mortality due to sepsis, severe sepsis and septic shock in Italian Pediatric Intensive Care Units: a prospective national survey. Intensive Care Med 2008; 34: 1690-7.

23.- Booy R, Habibi P, Nadel J, de Munter C, Britto J, Morrison A, et al. Reduction in case fatality rate from meningococcal disease associated with health care delivery. Arch Dis Child 2001; 85: 386-90.

24.- Carcillo J A, Tasker R C. Fluid resuscitation of hypovolemic shock: acute medicine's great triumph for children. Intensive Care Med 2006; 32: 958-61.

25.- Kumar A, Roberts D, Wood K E, Light B, Parrillo J E, Sharma S, et al. Duration of hypotension before initiation of effective antimicrobial therapy is the critical determinant of survival in human septic shock. Crit Care Med 2006; 34: 1589-96.

26.- Han Y Y, Carcillo J A, Dragotta M A, 
Bills D M, Watson R S, Westerman M E, et al. Early reversal of pediatric-neonatal septic shock by community physicians is associated with improved outcome. Pediatrics 2003; 112: 793-9.

27.- Hotchkiss, Karl I. The pathophysiology and treatment of sepsis. N Engl J Med 2003; 348: 138-50.

28.- Opal SM. Can we RESOLVE the treatment of sepsis?. Lancet 2007: 369; 803-4.

29.- Fiser R T, West N K, Bush A J, Sillos E M, Schmidt J E, Tamburro R F. Outcome of severe sepsis in pediatric oncology patients. Pediatr Crit Care Med 2005; 6: 531-6.

30.- Sorensen I A, Nielsen G G, Andersen P K, Teasdale T W. Genetic and environmental influences on premature death in adults adoptees. N Engl J Med 1988; 318: 727-32.

31.- Fijen C A, Derkx B H, Kuijper E J, Mannens M, Poort S R, Peters M, et al. Fulminant meningococcal septic shock in a boy with combined inherited properdin and protein $\mathrm{C}$ deficiency. Clin Exp Immunol 1995; 102: 290-6.

32.- Cohen J, Cristofaro P, Carlet J, Opal S M. New method of classifying infections in critically ill patients. Crit Care Med 2004;32: 1510-26.

33.- Reyes W J, Brimioulle S, Vincent J L. Septic shock without documented infection: an uncommon entity with a high mortality. Intensive Care Med 1999; 25: 1267-70.

34.- Renaud B, Brun-Buisson C, ICU-Bacteremia
Study Group. Outcomes of primary and catheter-related bacteremia. A cohort and case-control study in critically ill patients. Am J Respir Crit Care Med 2001; 163: 1584-90.

35.- Alberti C, Brun-Buisson C, Chevret S, Antonelli M, Goodman S V, Martin C, et al. Systemic inflammatory response and progression to severe sepsis in critically ill infected patients. Am J Respir Crit Care Med 2005; 171 : 461-8.

36.- Abraham, Singer M. Mechanisms of sepsisinduced organ dysfunction. Crit Care Med 2007; 35: 2408-16.

37.- Meisner. Biomarkers of sepsis: clinically useful? Curr Opin Crit Care 2005; 11: 473-80.

38.- Viallon A, Guyomarc'h S, Marjollet O, Berger C, Carricajo A, Robert F, et al. Can emergency physicians identify a high mortality subgroup of patients with sepsis: role of procalcitonin. Eur J Emerg Med 2008; 15: 26-33.

39.- Luzzani A, Polati E, Dorizzi R, Rungatscher A, Pavan R, Merlini A. Comparison of procalcitonin and $\mathrm{C}$-reactive protein as markers of sepsis. Crit Care Med 2003; 31: 1737-41.

40.- Wong H, Cvijanovich N, Wheeler D, Bigham M T, Monaco M, Odoms K, et al. Interleukin-8 as a stratification tool for interventional trials involving pediatric septic shock. Am J Respir Crit Care Med 2008; 178: 276-82.
41.- Samransamruajkit R, Heranrat T, Praphal N, Sritippayawan S, Deerojanawong J, Poovorawan Y. Levels of protein $\mathrm{C}$ activity and clinical factors In early phase of pediatric septic shock may be associated with the risk of death. Shock 2007; 28: 518-23.

42.- Venkataseshan S, Dutta S, Ahluwalia J, Narang A. Low plasma protein $\mathrm{C}$ values predict mortality in low birth weight neonates with septicemia. Pediatr Infect Dis J 2007; 26: 684-8.

43.- Donoso A, León J, Rojas G, Ramírez M, Oberpaur B. Valor de la leucopenia durante la primera hora como marcador pronóstico en la enfermedad meningocóccica. Rev Chil Pediatr 2004; 75: 441-7.

44.- Leclerc F, Leteurtre S, Duhamel A, Grandbastien B, Proulx F, Martinot A, et al. Cumulative influence of organ dysfunctions and septic state on mortality of critically ill children. Am J Respir Crit Care Med 2005; 171: 348-53.

45.- Dalton. Recombinant activated protein $\mathrm{C}$ in pediatric sepsis. Pediatr Infect Dis J 2003; 22: 743-5.

46.- van der Poll T. Immunotherapy of sepsis. Lancet Infect Dis 2001; 1: 165-74.

47.- Rubulotta F, Marshall J C, Ramsay G, Nelson D, Levy M, Williams M. Predisposition, insult/infection, response, and organ dysfunction: A new model for staging severe sepsis. Crit Care Med 2009; 37: 1329-35. 